Pacific Journal of Mathematics

APPLICATIONS OF THE SUBORDINATION PRINCIPLE TO 


\section{APPLICATIONS OF THE SUBORDINATION \\ PRINCIPLE TO UNIVALENT FUNCTIONS}

M. S. ROBERTSON

1. Introduction. Let

$$
f(z)=z+a_{2} z^{2}+\cdots+a_{n} z^{n}+\cdots
$$

be regular and univalent in $|z|<1$ and map $|z|<1$ onto a simplyconnected domain $D$. Let

$$
\phi(z)=b_{1} z+b_{2} z^{2}+\cdots+b_{n} z^{n}+\cdots
$$

also be regular in $|z|<1 . \phi(z)$ is said to be subordinate to $f(z)$ if for each $z$ of the unit circle $|z|<1$ the corresponding point $w=\phi(z)$ lies in the domain $D$. In this case [2] there exists an analytic function $\omega(z)$ regular in $|z|<1$ for which $\omega(0)=0,|\omega(z)| \leqq|z|<1$ and $\phi(z) \equiv$ $f\{\omega(z)\}$.

It is the purpose of this paper to establish the following basic Theorems $\mathrm{A}$ and $\mathrm{B}$ which concern analytic functions $F(z, t)$ and $\omega(z, t)$, depending upon a real parameter $t$, and then to use them to obtain results in the theory of univalent functions. Some of the results are well known and others are new, but the method of attack seems to be novel, simple and of sufficient generality to be of interest in itself. The functions $F(z, t)$ and $\omega(z, t)$ will be related to the univalent function $f(z)$ of (1.1) by means of the subordination concept.

An interesting biproduct of Theorem $B$ is the following statement. A sufficient condition that $f(z)$, regular and univalent in $|z|<1$, be convex in $|z|<1$ is that the de la Vallée Poussin means $V_{n}(z)$ of (1.1) be subordinate to $f(z)$ in $|z|<1$ for $n=1,2, \cdots$. Recently [3] G. Pólya and I. J. Schoenberg showed that this condition for convexity is also necessary.

TheOREM A. Let

$$
\omega(z, t)=\sum_{1}^{\infty} b_{n}(t) z^{n}
$$

be regular in $|z|<1$ for $0 \leqq t \leqq 1$. Let

$$
|\omega(z, t)|<1 \text { for }|z|<1,0 \leqq t \leqq 1, \omega(z, 0) \equiv z .
$$

Let $\rho$ be a positive real number for which

$$
\omega(z)=\lim _{t \rightarrow 0+}\left\{\frac{\omega(z, t)-z}{z t^{\rho}}\right\}
$$

Received October 2, 1959. 
exists. Then

$$
\mathscr{R} \omega(z) \leqq 0 \text { for }|z|<1 \text {. }
$$

If $\omega(z)$ is also analytic in $|z|<1$ and $\mathscr{R} \omega(0) \neq 0$, then

$$
\mathscr{R} \omega(z)<0 \text { for }|z|<1 \text {. }
$$

Proof. By Schwarz' lemma we have for $|z|<1|\omega(z, t)| \leqq|z|$ with equality only if $\omega(z, t)=z \exp i \theta(t)$, then the function

$$
\mu(z, t)=\frac{\omega(z, t)-z}{\omega(z, t)+z}
$$

is regular and $\mathscr{R} \mu(z, t)<0$ for $|z|<1$. But when $\omega(z, t)=z \exp i \theta(t)$, $\mu(z, t)=i \tan (1 / 2 \theta(t))$ is purely imaginary. Thus $\mu(z, t)$ is regular and $\mathscr{R} \mu(z, t) \leqq 0$ in $|z|<1$ with equality occurring only if $\omega(z, t)=z \exp i \theta(t)$. For $t>0,|z|<1$ we may write

$$
\mathscr{R}\left\{\frac{\omega(z, t)-z}{z t^{\rho}} \cdot \frac{2 z}{\omega(z, t)+z}\right\}=\mathscr{R}\left\{\frac{2 \mu(z, t)}{t^{\rho}}\right\} \leqq 0 .
$$

(1.4) implies that $\lim _{t \rightarrow 0+} \omega(z, t)=z=\omega(z, 0)$. Therefore, on letting $t \rightarrow 0$ in (1.7) we obtain $\mathscr{R} \omega(z) \leqq 0$ for $|z|<1$. When $\omega(z)$ is also analytic in $|z|<1$ and $\mathscr{R} \omega(0) \neq 0$ we have further that $\mathscr{R} \omega(z)<0$ in $|z|<1$. This follows since the maximum, in this case zero, of a non-constant harmonic function cannot occur at an interior point.

As an illustration of Theorem A, the following example is useful. Let

$$
\omega(z, t)=\frac{(1-2 t) z+z^{2}}{1+(1-2 t) z}, \quad 0 \leqq t \leqq 1 .
$$

Then $\omega(z, 0) \equiv z,|\omega(z, t)| \leqq 1$ in $|z|<1,0 \leqq t \leqq 1$.

$$
\begin{gathered}
\omega(z)=\lim _{t \rightarrow 0}\left\{\frac{\omega(z, t)-\omega(z, 0)}{z t}\right\}=\frac{1}{z}\left[\frac{\partial}{\partial t} \omega(z, t)\right]_{t=0}=2 \frac{z-1}{z+1}, \\
\mathscr{R} \omega(z)=2 \mathscr{R}\left(\frac{z-1}{z+1}\right)<0, \quad|z|<1 .
\end{gathered}
$$

Theorem A is a special case of Theorem B to follow. However, the proof of Theorem B depends upon Theorem A.

THEOREM B. Let

$$
f(z)=z+a_{2} z^{2}+\cdots+a_{n} z^{n}+\cdots
$$

be regular and univalent in $|z|<1$. For $0 \leqq t \leqq 1$ let $F(z, t)$ be regular in $|z|<1$. Let $F(z, 0) \equiv f(z)$ and $F(0, t) \equiv 0$ : Let $\rho$ be a positive 
real number for which

$$
F(z)=\lim _{t \rightarrow 0+}\left\{\frac{F(z, t)-F(z, 0)}{z t^{\rho}}\right\}
$$

exists. Let $F(z, t)$ be subordinate to $f(z)$ in $|z|<1$ for $0 \leqq t \leqq 1$. Then

$$
\mathscr{R}\left\{\frac{F(z)}{f^{\prime}(z)}\right\} \leqq 0,|z|<1
$$

If in addition $F(z)$ is also analytic in $|z|<1$ and $\mathscr{R} F(0) \neq 0$, then

$$
\mathscr{R}\left\{\frac{f^{\prime}(z)}{F(z)}\right\}<0, \quad|z|<1 \text {. }
$$

Proof. Since $F(z, t)$ is subordinate to $f(z)$ in $|z|<1$ we have

$$
F(z, t)=f\{\omega(z, t)\}, \quad|z|<1, \quad 0 \leqq t \leqq 1,
$$

where $\omega(z, t)$ is regular and bounded $|\omega(z, t)| \leqq 1$ in $|z|<1,0 \leqq t \leqq 1$. Since $F(z, 0) \equiv f(z)$ and since $f(z)$ is univalent in $|z|<1$ we have $\omega(z, 0) \equiv z$. Also since $f(0)=0, F(0, t)=0$ and since $f(z)$ is univalent we have $\omega(0, t)=0$. We now write

$$
\frac{F(z, t)-F(z, 0)}{z t^{\rho}}=\left[\frac{f(\omega(z, t))-f(\omega(z, 0))}{\omega(z, t)-\omega(z, 0)}\right]\left[\frac{\omega(z, t)-\omega(z, 0)}{z t^{\rho}}\right] .
$$

(1.12) implies that $F(z, t)$ is continuous from the right at $t=0$ and a similar statement holds for $\omega(z, t)$ because of the subordination. Let $t \rightarrow 0+$ in (1.14). The left side of equation (1.14) has for a limit $F(z)$ by (1.12). On the right side of (1.14) the square bracket has a limit $f^{\prime}(z) \neq 0$. Thus

$$
\omega(z)=\lim _{t \rightarrow 0+}\left[\frac{\omega(z, t)-\omega(z, 0)}{z t^{\rho}}\right]
$$

exists and equals $F(z) / f^{\prime}(z)$. Furthermore $\mathscr{R} \omega(0)=\mathscr{R} F(0)$. If $F(z)$ is analytic so is $\omega(z)$. Since the conditions of Theorem A are fulfilled by $\omega(z, t)$ we have

$$
\mathscr{R} \frac{F(z)}{f^{\prime}(z)}=\mathscr{R} \omega(z) \leqq 0, \quad|z|<1 .
$$

When $F(z)$ is analytic in $|z|<1$ and $\mathscr{R} F(0) \neq 0$ we also have

$$
\mathscr{R}\left\{\frac{f^{\prime}(z)}{F(z)}\right\}<0, \quad|z|<1 \text {. }
$$

2. Applications to univalent functions. The properties of univalent 
functions $W=f(z)$, given by (1.1), which are also star-like with respect to the origin in $|z|<1$ are well-known [2]. If $W=f(z)$ maps $|z|<1$ onto a star-like domain $D$ of the $W$-plane, then by definition the line segment joining the origin to the point $W=f(z)$ lines entirely within $D$ for each $z$ in $|z|<1$. One then shows that it is necessary that

$$
\mathscr{R}\left\{\frac{z f^{\prime}(z)}{f(z)}\right\}>0 \text { in }|z|<1 .
$$

In establishing (2.1) one is obliged to show first that if $f(z)$ is star-like with respect to the unit circle it is also star-like with respect to each smaller circle $|z|=r<1$. At this stage one then appeals to an alternative definition of a star-like domain.' This requires that the radius vector, joining the origin to the point $f(z)$, turns always in one direction as the argument of $z$ advances.

A much simpler proof of the necessity of (2.1) follows immediately from Theorem B. Since $(1-t) f(z)$ is subordinate to $f(z)$ for $0 \leqq t \leqq 1$, we have

$$
(1-t) f(z)=f\{\omega(z, t)\}
$$

where $\omega(z, t)$ satisfies the conditions of Theorem A. Taking $\rho=1$ and letting

$$
F(z, t)=(1-t) f(z)
$$

in Theorem B we obtain at once $F(z)=-f(z) / z \neq 0$, so that (2.1) follows from (1.17) very simply.

More generally we have the following theorem.

THEOREM 1. Let.

$$
f(z)=z+a_{2} z^{2}+\cdots+a_{n} z^{n}+\cdots
$$

be regular and univalent in $|z|<1$ and such that $\left(1-t e^{i \alpha}\right) f(z)$ is subordinate to $f(z)$ in $|z|<1$ for an interval $0 \leqq t \leqq t_{0}$, $\alpha$ a real constant $|\alpha|<\pi / 2$, then

$$
\mathscr{R}\left\{e^{-i \alpha} \frac{z f^{\prime}(z)}{f(z)}\right\}>0,|z|<1
$$

For the proof of Theorem 1 we take

$$
F(z, t)=\left(1-t e^{i \alpha}\right) f(z)
$$

in Theorem B and (1.13) becomes (2.4) in this case. The condition (2.4) is the one given for spiral-like functions by L. Špaček [7].

The following theorem from an intuitive point of view appears to be almost self-evident. Our new technique, however, furnishes an easy 
and precise proof.

THEOREM 2. Let $f(z)$ of (1.1) be regular and univalent in $|z|<1$. For an interval $0 \leqq t \leqq t_{0}$ let the function

$$
\frac{1}{2}\left[f\left(e^{i t} z\right)+f\left(e^{-i t} z\right)\right]
$$

be subordinate to $f(z)$ in $|z|<1$. Then.

$$
\mathscr{R}\left\{1+\frac{z f^{\prime \prime}(z)}{f^{\prime}(z)}\right\}>0, \quad|z|<1,
$$

and $f(z)$ is convex in $|z|<1$.

Proof. In Theorem B we choose $\rho=2$ and $F(z, t)$ to be the function (2.6). Then

$$
\begin{aligned}
& F(z)=\lim _{t \rightarrow 0} \frac{F(z, t)-F(z, 0)}{z t^{2}}=\lim _{t \rightarrow 0} \frac{1}{2 z t} \frac{\partial F(z, t)}{\partial t} \\
& F(z)=\lim _{t \rightarrow 0} \frac{1}{2 z} \frac{\partial^{2} F(z, t)}{\partial t^{2}}=-z f^{\prime \prime}(z)-f^{\prime}(z) .
\end{aligned}
$$

Since $f^{\prime}(0)=1$, it follows that $F(0)=-1$ so that $\mathscr{R} F(0) \neq 0$. Thus (1.17) of Theorem $B$ is equivalent to

$$
\mathscr{R}\left\{1+z \frac{f^{\prime \prime}(z)}{f^{\prime}(z)}\right\}>0, \quad|z|<1 .
$$

It is well known [2] that (2.9) implies that $f(z)$ is convex in $|z|<1$.

For odd functions and an appropriate choice of $F(z, t)$ we obtain a result perhaps not so intuitively obvious as Theorem 2 . It is the following theorem.

\section{TheOREM 3. Let}

$$
f(z)=z+\sum_{2}^{\infty} a_{2 n-1} z^{2 n-1}
$$

be an odd function, regular and univalent in $|z|<1$. For all real $\alpha$ and for an interval $0 \leqq t \leqq t_{0}$ let the function

$$
\frac{1}{2}\left[f\left(\frac{z+x}{1+\bar{x} z}\right)+f\left(\frac{z-x}{1-\bar{x} z}\right)\right], \quad x=t e^{i \alpha},
$$

be subordinate to $f(z)$ in $|z|<1$. Then $f(z)$ is convex in $|z|<1$.

For the proof of Theorem 3 we take $F(z, t)$ of Theorem B to be the function (2.11) and select $\rho=2$. A calculation of $F(z)$ in (1.12), 
together with (1.16), leads to the inequality

$$
\mathscr{R}\left[\left(1-e^{-2 i \alpha} z^{2}\right)^{2} e^{2 i \alpha} \frac{f^{\prime \prime}(z)}{z f^{\prime}(z)}-2\left(1-e^{-2 i \alpha} z^{2}\right)\right] \leqq 0,|z|<1 .
$$

Choose $\alpha=\operatorname{amp} z$. Let $|z|=r<1$. Then (2.12) becomes

$$
\begin{aligned}
& \mathscr{R}\left[\left(1-r^{2}\right)^{2} \cdot \frac{z f^{\prime \prime}(z)}{r^{2} f^{\prime}(z)}-2\left(1-r^{2}\right)\right] \leqq 0, \\
& \mathscr{R}\left[1+\frac{z f^{\prime \prime}(z)}{f^{\prime}(z)}\right] \leqq \frac{1+r^{2}}{1-r^{2}} .
\end{aligned}
$$

Similarly, for $\alpha=\pi / 2+\operatorname{amp} z$, we obtain

$$
\mathscr{R}\left[1+\frac{z f^{\prime \prime}(z)}{f^{\prime}(z)}\right] \geqq \frac{1-r^{2}}{1+r^{2}}>0 .
$$

It follows from (2.15) that $f(z)$ is convex for $|z|<1$. It is to be noticed that equality occurs in (2.13) for the convex function

$$
f(z)=\frac{1}{2} \nu \cdot \log \left(\frac{1+z}{1-z}\right) .
$$

when $\alpha=0$. In this case $F(z) \equiv 0$.

For another application of Theorem $B$ we turn now to a class of function which need not be convex but which form a subclass of the class of close-to-convex functions introduced by W. Kaplan [1].

It is well known that if

$$
f(z)=z+\sum_{2}^{\infty} a_{n} z^{n}
$$

is univalent and convex in $|z|<1$, then $\left|a_{n}\right| \leqq 1$ [2]. The author [5, 6] has shown that if the coefficients are all real and if $f(z)$ is univalent and convex only in the direction of the imaginary axis for $|z|<1$, then again $\left|a_{n}\right| \leqq 1$, but that if the coefficients are complex the results $\left|a_{n}\right| \leqq n$ is sharp. For the class of functions $f(z)$ which are close-toconvex in $|z|<1$, the inequalities $\left|a_{n}\right| \leqq n$ again hold [4]. We now consider another class of functions, which are also close-to-convex in $|z|<1$, but not necessarily convex, for which $\left|a_{n}\right| \leqq 1$. This class contains the odd star-like functions as a sub-class. The result is stated in the following theorem.

THEOREM 4. Let the function

$$
(1-t) f(z)+t f(-z)
$$

be subordinate to the univalent, regular function 


$$
f(z)=z+a_{2} z^{2}+\cdots+a_{n} z^{n}+\cdots
$$

in $|z|<1$ for an interval $0 \leqq t \leqq t_{0}$. Then

$$
\mathscr{R}\left\{\frac{z f^{\prime}(z)}{f(z)-f(-z)}\right\}>0,|z|<1,
$$

and the vector $\{f(z)-f(-z)\}$ turns continuously in one direction as $z$ traverses each circle $|z|=r<1 . \quad f(z)$ is close-to-convex in $|z|<1$.

Proof. Let $\rho=1$ and let $F(z, t)$ be the function in (2.17). Then $F(z)$ of (1.12) reduces to $(1 / z)[f(-z)-f(z)]$ and $F(0) \neq 0$. (1.13) then leads to (2.19).

Now let

$$
\begin{aligned}
\arg [f(z)-f(-z)]=\phi, \arg z=\theta . \\
\frac{d \phi}{d \theta}=\mathscr{R} z\left\{\frac{f^{\prime}(z)+f^{\prime}(-z)}{f(z)-f(-z)}\right\} \\
=\mathscr{R}\left\{\frac{z f^{\prime}(z)}{f(z)-f(-z)}\right\}+\mathscr{R}\left\{\frac{(-z) f^{\prime}(-z)}{f(-z)-f(z)}\right\}>0,|z|<1,
\end{aligned}
$$

by $(2.19)$.

Since by (2.20) $\{f(z)-f(-z)\}$ is univalent and star-like in $|z|<1$, it follows that

$$
\psi(z)=\int_{0}^{z} \frac{f(t)-f(-t)}{t} d t, \quad|z|<1
$$

is convex in $|z|<1$. Thus (2.19) may be cast in the form

$$
\mathscr{R}\left\{\frac{f^{\prime}(z)}{\psi^{\prime}(z)}\right\}>0, \quad|z|<1, \quad \varphi(z) \text { convex, }
$$

which implies that $f(z)$ is close-to-convex [1] in $|z|<1$. This completes the proof of Theorem 4 .

In a recent paper [3] G. Pólya and I. J. Schoenberg have shown that if $f(z)$ of (1.1) is univalent and convex in $|z|<1$ then so are the de la Vallée Poussin means $V_{n}(z)$ of the power series (1.1),

$$
\begin{aligned}
V_{n}(z)= & \frac{n}{n+1} z+\frac{n(n-1)}{(n+1)(n+2)} a_{2} z^{2}+\cdots \\
& +\frac{n(n-1) \cdots 1}{(n+1)(n+2) \cdots(2 n)} a_{n} z^{n},
\end{aligned}
$$

and if $D$ and $D_{n}$ denote the convex domains into which the unit circle is mapped by $f(z)$ and $V_{n}(z)$, respectively, then $D_{n} \subset D$. In other words, $V_{n}(z)$ is necessarily subordinate to $f(z)$ for $n=1,2, \cdots$ when $f(z)$ is 
univalently convex. By means of Theorem $B$ we can now prove that the condition $D_{n} \subset D$ for infinitely many values of $n$ is also a sufficient condition that $f(z)$ be convex when $f(z)$ is univalent. The theorem of Pólya and Schoenberg in its extended form is now stated as Theorem 5.

THEOREM 5. A necessary and sufficient condition that the function

$$
f(z)=z+a_{2} z^{2}+\cdots+a_{n} z^{n}+\cdots,
$$

regular and univalent in $|z|<1$, be convex in $|z|<1$ is that the de la Vallée Poussin means $V_{n}(z)$ in (2.23) be subordinate to $f(z)$ in $|z|<1$ for $n=1,2, \cdots$.

Proof of sufficiency. In Theorem B we choose $\rho=1$ and $F(z, t)=$ $V_{n}(z) \quad$ where $t=(n+1)^{-1}$. We define $F(z, 0)=\lim _{t \rightarrow 0+} F(z, t)=$ $\lim _{n \rightarrow \infty} V_{n}(z)=f(z)$, uniformly in $|z| \leqq r<1$. For $\rho=1$ we shall show that the limit defining $F(z)$ in (1.12) exists uniformly and is precisely the analytic function $-\left\{z f^{\prime \prime}(z)+f^{\prime}(z)\right\}, F(0)=-1$. When this is done (1.17) will give

$$
\mathscr{R}\left\{1+\frac{z f^{\prime \prime}(z)}{f^{\prime}(z)}\right\}>0, \quad|z|<1,
$$

and the convexity of $f(z)$ follows. We need the following lemma.

LEMMA. If $n$ and $k$ are positive integers, $k \leqq n$, then

$$
(n+1)\left[1-\frac{n(n-1) \cdots(n-k+1)}{(n+1)(n+2) \cdots(n+k)}\right] \leqq k^{2} .
$$

We establish the lemma by mathematical induction. Let $n$ be an assigned positive integer. It is readily seen that (2.24) holds for $k=1$. Assuming that (2.24) is true for a value $k<n$ we prove that (2.24) also holds when $k$ is replaced by $(k+1)$. Indeed, we have

$$
\begin{aligned}
(n+1) & {\left[1-\frac{n(n-1) \cdots(n-k+1)(n-k)}{(n+1)(n+2) \cdots(n+k)(n+k+1)}\right] } \\
= & (n+1)\left[1-\frac{n(n-1) \cdots(n-k+1)}{(n+1)(n+2) \cdots(n+k)}\left(1-\frac{2 k+1}{n+k+1}\right)\right] \\
& =(n+1)\left[1-\frac{n(n-1) \cdots(n-k+1)}{(n+1)(n+2) \cdots(n+k)}\right] \\
& +(2 k+1) \frac{(n+1)}{n+k+1}\left[\frac{n(n-1) \cdots(n-k+1)}{(n+1)(n+2) \cdots(n+k)}\right] \\
& \leqq \\
& k^{2}+(2 k+1)=(k+1)^{2} .
\end{aligned}
$$


Turning to the calculation of $F(z)$ we have

$$
\begin{aligned}
F(z)= & \lim _{t \rightarrow 0+}\left[\frac{F(z, t)-F(z, 0)}{t z}\right]=\lim _{n \rightarrow \infty} \frac{n+1}{z}\left(V_{n}(z)-f(z)\right) \\
= & -\lim _{n \rightarrow \infty}(n+1) \sum_{k=1}^{n}\left\{1-\frac{n(n-1) \cdots(n-k+1)}{(n+1)(n+2) \cdots(n+k)}\right\} a_{k} z^{k-1}, a_{1}=1, \\
& -\lim _{n \rightarrow \infty}(n+1) z^{n} \sum_{\nu=0}^{\infty} a_{\nu+n+1} z^{\nu} .
\end{aligned}
$$

Let $|z| \leqq r<1$. Since $f(z)$ is univalent we have $\left|a_{\nu+n+1}\right|<e(\nu+n+1)$. Consequently for large $n$

$$
\begin{gathered}
(n+1) z^{n} \sum_{\nu=0}^{\infty} \alpha_{\nu+n+1} z^{\nu}=0\left\{\frac{n^{2} r^{n}}{(1-r)^{2}}\right\}=p_{n}, \\
\lim p_{n}=0
\end{gathered}
$$

uniformly in $|z| \leqq r$.

Let $N$ be a positive integer. Then

$$
\begin{aligned}
\lim _{n \rightarrow \infty}(n+ & 1) \sum_{k=1}^{N}\left\{1-\frac{n(n-1) \cdots(n-k+1)}{(n+1)(n+2) \cdots(n+k)}\right\} a_{k} z^{k-1} \\
& =\sum_{k=1}^{N} k^{2} a_{k} z^{k-1} .
\end{aligned}
$$

For $n>N,|z| \leqq r$, by the lemma we have

$$
\begin{aligned}
& \left|(n+1) \sum_{k=N+1}^{n}\left\{1-\frac{n(n-1) \cdots(n-k+1)}{(n+1)(n+2) \cdots(n+k)}\right\} a_{k} z^{k-1}\right| \\
& \leqq \\
& \quad \sum_{k=N+1}^{n} k^{2}\left|a_{k}\right| r^{k-1}<e \sum_{N+1}^{\infty} k^{3} r^{k-1} .
\end{aligned}
$$

Given $\varepsilon>0$, we now choose $N_{0}(\varepsilon, r)$ so that for $N>N_{0}$

$$
e \sum_{N+1}^{\infty} k^{3} r^{k-1}<\varepsilon
$$

From (2.26), (2.28), (2.29), (2.30) and (2.31) it follows that the limit in (2.26) exists uniformly in $|z| \leqq r<1$ and is the analytic function

$$
\begin{aligned}
F(z) & =-\sum_{k=1}^{\infty} k^{2} a_{k} z^{k-1}, a_{1}=1,|z|<1, \\
& =-z f^{\prime \prime}(z)-f^{\prime}(z)
\end{aligned}
$$

This completes the proof of the sufficiency part of Theorem 5. The necessity part was shown in [3]. In (2.26) since $n$ is a positive integer we have let $t \rightarrow 0$ through a discrete set of values of $t$. This, however, in no way affects the validity of Theorem $B$. 


\section{REFERENCES}

1. W. Kaplan, Close-to-convex Schlicht functions, Michigan Math. Journal, 1 (1952), 169185.

2. Z. Nehari, Conformal Mapping, New York, 1952.

3. G. Pólya and I. J. Schoenberg, Remarks on de la Vallée Poussin means and convex conformal maps of the circle, Pacific J. Math., 8 (1958), 295-334.

4. M. O. Reade, Sur une classe de fonctions univalentes, C. R. Acad. Sci. Paris, 239 (1954), 1758-1759.

5. M.S. Robertson, On the theory of univalent functions, Annals of Math., 37 (1936), 374-408.

6. - Analytic functions star-like in one direction, Amer. J. Math., 58 (1936), 465472.

7. L. Špaček, Contribution à la theorie des fonctions univalentes, Časopsis pro Pěstováni Matematiky a Fysiki, 62 (1932), 12-19.

RUtgers * The StATE UNIVERSity. 


\section{PACIFIC JOURNAL OF MATHEMATICS}

\section{EDITORS}

Ralph S. Phillips

Stanford University

Stanford, California

F. H. BrownelL

University of Washington

Seattle 5 , Washington
A. L. Whiteman

University of Southern California Los Angeles 7. California

L. J. PAIGE

University of California

Los Angeles 24, California

ASSOCIATE EDITORS
E. F. BECKENBACH
D. DERRY
H. L. ROYDEN
E. G. STRAUS
T. M. CHERRY
M. OHTSUKA
E. SPANIER
F. WOLF

\section{SUPPORTING INSTITUTIONS}

UNIVERSITY OF BRITISH COLUMBIA

CALIFORNIA INSTITUTE OF TECHNOLOGY

UNIVERSITY OF CALIFORNIA

MONTANA STATE UNIVERSITY

UNIVERSITY OF NEVADA

NEW MEXICO STATE UNIVERSITY

OREGON STATE COLLEGE

UNIVERSITY OF OREGON

OSAKA UNIVERSITY

UNIVERSITY OF SOUTHERN CALIFORNIA
STANFORD UNIVERSITY

UNIVERSITY OF TOKYO

UNIVERSITY OF UTAH

WASHINGTON STATE COLLEGE,

UNIVERSITY OF WASHINGTON

AMERICAN MATHEMATICAL SOCIETY

CALIFORNIA RESEARCH CORPORATION

HUGHES AIRCRAFT COMPANY

SPACE TECHNOLOGY LABORATORIES

NAVAL ORDNANCE TEST STATION

Printed in Japan by International Academic Printing Co., Ltd., Tokyo, Japan

Reprinted 1966 in the United States of America 


\section{Pacific Journal of Mathematics}

\section{Vol. 11, No. $1 \quad$ November, 1961}

A. A. Albert, Generalized twisted fields ............................ 1

Richard Arens, Operational calculus of linear relations ................... 9

John Herbert Barrett, Disconjugacy of a self-adjoint differential equation of the fourth order ....................................... 25

Paul Richard Beesack, Hardy's inequality and its extensions ............... 39

Julius Rubin Blum and David Lee Hanson, On invariant probability measures.

II .............................................

Robert Allen Bonic, Symmetry in group algebras of discrete groups.......... 73

R. Creighton Buck, Multiplication operators ...................... 95

Jack Gary Ceder, Some generalizations of metric spaces ................. 105

Meyer Dwass, Random crossings of cumulative distribution functions ......... 127

Albert Edrei, Wolfgang H. J. Fuchs and Simon Hellerstein, Radial distribution and

deficiencies of the values of a meromorphic function ............... 135

William Cassidy Fox, Harmonic functions with arbitrary local singularities ..... 153

Theodore Thomas Frankel, Manifolds with positive curvature ............... 165

Avner Friedman, A strong maximum principle for weakly subparabolic

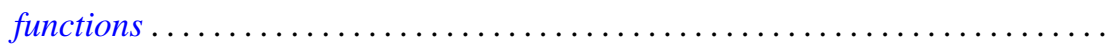

Watson Bryan Fulks and J. O. Sather, Asymptotics. II. Laplace's method for

multiple integrals ......................................

Adriano Mario Garsia and Eugene Richard Rodemich, An embedding of Riemann

surfaces of genus one ..................................... 193

Irving Leonard Glicksberg, Weak compactness and separate continuity......... 205

Branko Grünbaum, On a conjecture of H. Hadwiger .................. 215

Frank J. Hahn, On the action of a locally compact group on $E_{n} \ldots \ldots \ldots \ldots \ldots . . \ldots 221$

Magnus R. Hestenes, Relative hermitian matrices ..................... 225

G. K. Kalisch, On similarity invariants of certain operators in $L_{p} \ldots \ldots \ldots \ldots .247$

Yitzhak Katznelson and Walter Rudin, The Stone-Weierstrass property in Banach

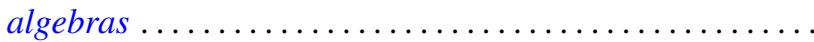

Samir A. Khabbaz, The subgroups of a divisible group $G$ which can be represented as intersections of divisible subgroups of $G \ldots \ldots \ldots \ldots \ldots \ldots \ldots \ldots \ldots . \ldots \ldots 7$

Marvin Isadore Knopp, Construction of a class of modular functions and forms .......................................... 275

Charles Alan McCarthy, Commuting Boolean algebras of projections .......... 295

T. M. MacRobert, Transformations of series of E-functions ................ 309

Heinz Renggli, An inequality for logarithmic capacities ................. 313

M. S. Robertson, Applications of the subordination principle to univalent functions .......................................... 315

David Sachs, Partition and modulated lattices ..................... 325

Frank S. Scalora, Abstract martingale convergence theorems ............... 347

Elbert A. Walker, Torsion endomorphic images of mixed Abelian groups ........ 375

Morgan Ward, The prime divisors of Fibonacci numbers................. 379

Charles R. B. Wright, On the nilpotency class of a group of exponent four....... 387 